\title{
Study on the Multi-domain Modeling and Optimization Designing Method for the Band Sawing Equipment
}

\author{
Guojin Chen, Jing Ni, Tingting Liu \\ School of Mechanical Engineering \\ Hangzhou Dianzi University \\ Hangzhou, 310018, China \\ chenguojin@163.com
}

\author{
Huipeng Chen, Ming Xu \\ School of Mechanical Engineering \\ Hangzhou Dianzi University \\ Hangzhou, 310018, China \\ cgj@hdu.edu.cn
}

\begin{abstract}
This paper is oriented to the design and analysis process of the high-level band sawing equipment, and studied the modeling and the simulated optimization design method for the high-level band sawing equipment based on the unified modeling language. The unified constraint model is used to describe the multi-domain model of the CNC band sawing equipment. The model libraries for the mechanical structure, the hydraulic pressure and the control of the CNC band sawing equipment are established to complete the unified multi-domain constraint model for the simulation optimization. Aiming at the multi-domain, multi-objective, multi-constraint and multi-variable characteristics for the model of the highlevel band sawing equipment, we studied the planning solution, the optimization strategy and the analysis methods for the multidisciplinary design optimization based on the multidomain model. The unified simulation platform for the multifield of the corresponding cutting equipment is developed to provide the research foundation and the technical support for optimizing and improving the accuracy and efficiency of the band sawing equipment.
\end{abstract}

Keywords- multi-domain modeling; multidisciplinary design optimization; band sawing equipment;CAD/CAM

\section{INTRODUCTION}

The band sawing equipment is a complex system of the mechanical-electric-hydraulic-control, and its design is a very difficult and complex task. While the present various engineering applications gradually improve the performance of the band saw equipment, the design of the band saw system is becoming increasingly complex and difficult. When the band saw equipment is developed today in China, the empirical design method is widely used. It is reliable in quality, but it is difficult for product innovation and technological innovation. The much material consumption, more complex structure, high cost and difficulty in the application of new technologies are increasingly unable to adapt to the applications of the modern engineering technology in the band saw equipment. How to successfully apply new technologies and to implement new design concepts for improving the technical level of the band saw equipment and developing the high-level band saw equipment becomes a major issue need to be solved. The multidisciplinary design optimization (MDO) is just the new design method of the complex system proposed to achieve better the new design concept. Its main idea is to fully utilize the distributed computer network technology to integrate the various disciplines of knowledge in the entire design process of the complex systems. In accordance with the designoriented thinking, the various disciplines of models and analytical tools are integrated. Through the effective design, the optimization of the strategy, and the management of the design process, the coordination interaction between the various disciplines is fully used to obtain the optimal solution of the overall system. On the foundation of the multidisciplinary design optimization method, the analytical tools are used to build the efficient design and analysis platform for the band saw equipment. So that can get the optimal design of the overall system. The design platform is of great significance for the innovative design of the band saw equipment, the applications of new technologies to improve the design efficiency, technical level and competitiveness of the band saw equipment, the reduction of costs and the shortening of the design cycle.

\section{BAND SAW EQUIPMENT's COMPONENTS}

The typical band saw equipment is shown in Figure 1, and is composed mainly by the gantry framework, the driving and driven saw disks, the band saw, the correcting device, the hydraulic feeding cylinder, the sawing workpiece, the sawing bench, the feeding guide post, the base, and the associated electrical control system. The sawing principle of the specific band saw equipment: the band saw blades are wound on the driving and driven saws discs and the correction device. The correction device guarantees that the band saw blades are parallel with the workpiece's cutting surface. The spacing between the driving and driven discs is adjusted by the tensioning hydraulic-cylinder to tension the saw blades. The main movement is generated by the driving pulley (driven by the variable frequency motor). The feeding movement is generated by the feeding hydraulic-cylinder in the vertical direction. Furthermore, in Figure 1, TM is the driven torque of the driving and driven saw discs. A, B, C, D are the tangent point's marking for the band saw blade and the band saw disc. O1, $\mathrm{O} 2$ are the axle center's marking for the driving and driven saw discs. T0 (s), T1 (s), T2 (s), T3 (s) are respectively for the tensions suffered on the band saw at A, B, C, D. $s$ is the coordinate variable of the arc length for the band saw blade, taking $\mathrm{A}$ as the origin. F0 is the tensioning force for the band saw blade. 


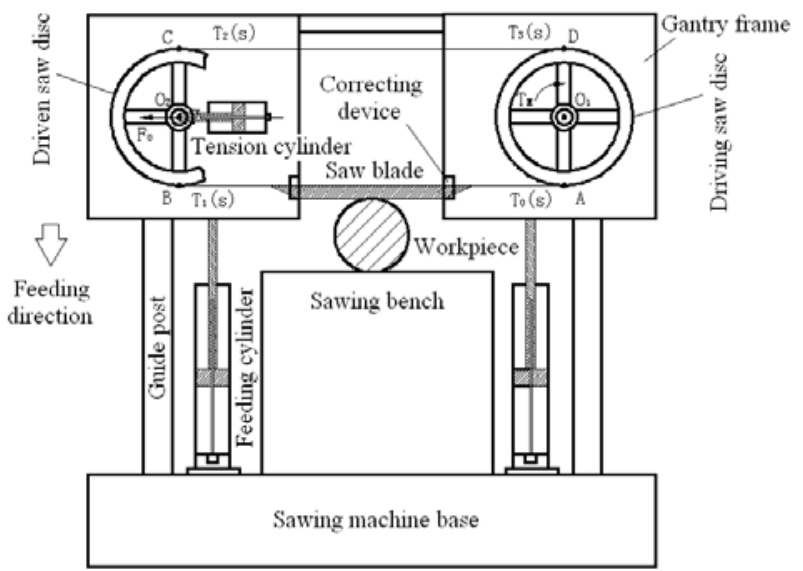

Figure 1. The typical band saw equipment

\section{Overall Program AND ARChITECTURE}

In this paper, under the unified framework platform, the integrated design environment of the multi-domain physical system's performance analysis and design for the multidisciplinary design optimization analysis is established, as shown in Figure 2. Each subsystem is interfaced with the international common system under the unified framework. Facing to the band saw equipment's design, the integrated environment of the performance analysis and the multidisciplinary design optimization for the multi-domain mixed system are realized. From the levels, the system can be divided into three logic levels, namely, the system support layer, the collaborative control layer, the enable tool layer.

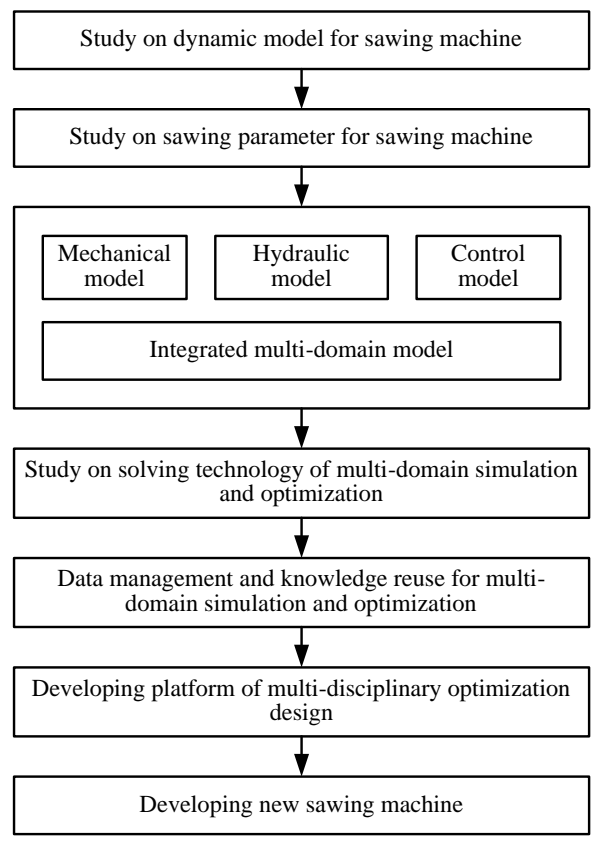

Figure 2. The integrated design environment
1) The system support layer: This layer is used to support the storage and management of the data and knowledge which is relevant with the product development, including the product structure data, the model data, the analysis and simulation data, the testing and trial data, the product development processes and specifications, the product design knowledge, and the other shared resource data.

2) The cooperative control layer: The cooperative control level studies and solves the collaborative design problem, facing to the cross-departmental and multidisciplinary design process of the complex products. Through the operating mechanism of the computer network support, the data flow engine, the workflow engine, the messaging engine, the knowledge inferring engine, the resource scheduling strategy, the data security management strategy, the collaborative conflict management strategies, the uniform code management strategy, and the distributed computing management strategy, the tasks of the demand analysis, the product design, the analysis simulation, the prototype testing, the manufacturing and the after-sale maintenance are managed co-operationally in the product development process, including the project development team management, the project task management, the collaborative task modeling, the workflow modeling, the design simulation, the process control, the collaborative message management, and the data collaboration management, etc.. The collaborative control layer is the core layer of the platform to be established, and is also the research focus.

3) The enable tool layer: This layer consists of three parts, namely, the enable tool set, the application tool set and the interface tool set. The enable tool (the system tool set in Figure) includes the application components in the collaborative platform. The application components realize the collaborative management of the design simulation, specifically including the management tools for the resources, data, knowledge, processes, etc. of the design simulation and the tools for the multi-domain simulation modeling, analysis simulation, visualization, and so on. The application tool set is mainly the unit application tools, such as the tools for product design, analysis simulation, auxiliary test and other aspects. The interface tool set offers mainly the interfaces for the unit application system integration, data security control, data format conversion, and function calling, and so on.

In the performance optimization analysis process for the digital functional prototype of the band saw equipment based on the multidisciplinary design optimization, first, the integrated environment interface operation is designed. The model units in the model unit library of the multi-domain physical system are used to establish the digitizing function prototype model. Then under the simulation solution control of the interface operation, the physical model of the digitizing function prototype is converted into the mathematical model and calls the multi-field solving engine for the adaptive solving. The result data generated by the solving engine retain in the result database which drive the digitizing function prototype to carry out the high-speed animation simulation or visualization data display, or as a basis for optimization design. 
IV. Multi-domain Integrated Modeling System AND SiMULATION OPTIMIZATION SOLUTION OF BAND SAW EQUIPMENT

The multi-domain physical mixing system of the highlevel band saw equipment is composed by the multi-domain physical components, such as mechanical, electrical, fluid, control. In order to solve the analysis design and performance simulation, three architectures can be used.

1) The virtual prototype system at the core of the traditional mechanical system is extended so that it can process the mixed problem of the mechanical, hydraulic and control.

2) The multi-domain physical system is directly taken as the research object. The machinery is one of the many fields. The various fields of mechanical, electrical, hydraulic, control are equally treated.

3) The multi-domain physical system and the mechanical-core virtual function prototype system are realized respectively. And their frameworks are integrated.

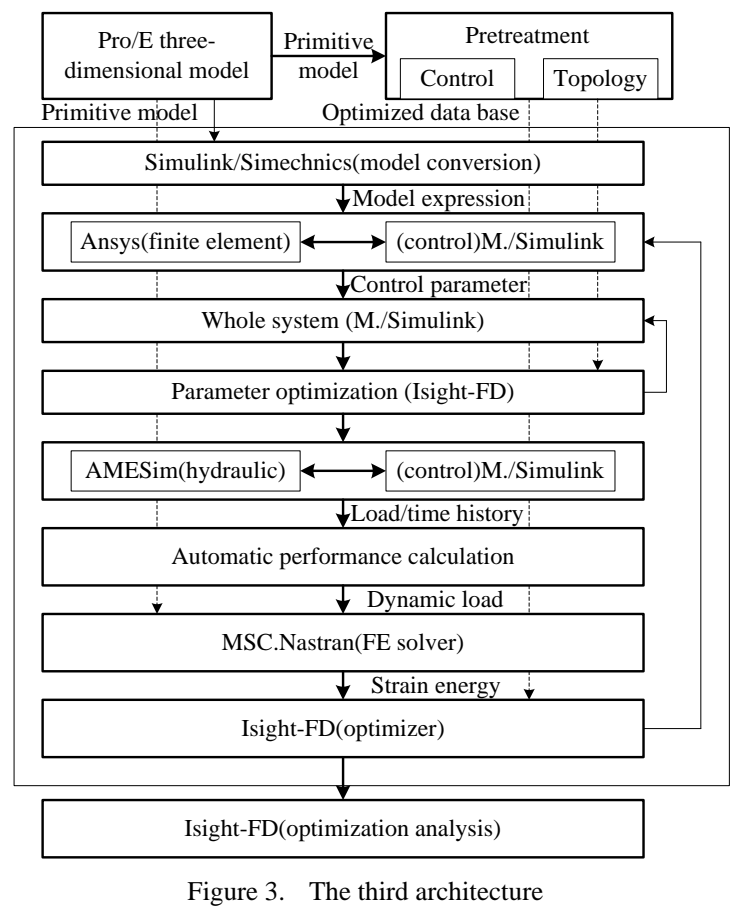

The first architecture is the expansion of the electrical, hydraulic and control fields in the mechanical-core virtual prototype system. The structure has been largely restricted, and the expansion of the multi-domain physical model units is of the poor reusability. The second architecture is the equal modeling for the each functional unit of the multi-domain physical systems, and the unified solution. But it can only treat the rigid-flexible hybrid multi-body system problems, and can not directly reuse the structural design results of the mechanical systems. The third architecture can respectively realize the multi-domain physical systems and the machinery-core virtual function prototype system and integrate their frameworks. That can not only achieve the digitized functional prototype system integrated with CAD/CAM, and can take advantage of the multi-domain physical subsystem platform to make the digitized functional prototype be of the multi-field scalability. The expansion on the basis has good reusability. We use the third architecture, as shown in Figure 3.

\section{MULTIDISCIPLINARY DESIGN OPTIMIZATION AND ANALYSIS PLATFORM FOR BAND SAW EQUIPMENT}

On the basis of the multi-domain simulation model of the high-level band saw equipment, the multidisciplinary design optimization is done to obtain the optimal overall system performance.

The research object of MDO is the design optimization problem of the complex products. First, the design optimization problems of the complex products are accurately expressed at the precise mathematical description, namely, the mathematical modeling of the MDO problems. The general mathematical expression is as follows.

$$
\begin{aligned}
\text { Find } & : Z, Y, X, i, j=1 \cdots n, i \neq j \\
\text { Min } & : F=f_{o}\left(Z, Y_{o}, Y\right) \\
\text { Satisfy } & : E_{0}\left(Z, Y_{o}, Y_{i}\right)=0, E_{i}\left(Z, X_{i}, Y_{j i}, Y_{i}\right)=0 \quad, \quad X=\left(\begin{array}{c}
X_{1} \\
\vdots \\
X_{n}
\end{array}\right) \\
\text { s.t. }: & G_{o} \leq 0, G_{i} \leq 0
\end{aligned}
$$

In the formula, $\mathrm{Z}$ is the system design variable, $\mathrm{Y}$ is the system state variable, $\mathrm{X}$ is the disciplinary design variable, $\mathrm{E} 0$ is the system state equation, Ei is the disciplinary state equation, G0 is the system inequality constraint set, Gi is the disciplinary inequality constraint set. MDO is making the objective function $\mathrm{F}$ to be the maximum or minimum. The mathematical model equation determines the coupling relationship of the disciplinary analysis and the interdiscipline. The state equation E0, Ei is generally described at the coupled differential equations.

Because the multi-disciplinary model has the complex cross-coupled relationship, the system is generally decomposed during the MDO analysis. The decomposition of the system is that the entire system is decomposed into the multiple independent subsystems in accordance with the certain rules. Decomposition of the system can not only improve the optimization efficiency. And it is more important to make the original problem to simplify. That can meet the required optimization results. The key of the system decomposition is to decompose and re-plan the system in accordance with the contained disciplines (subsystems) and the certain rules on the premise of ensuring the overall system performance optimal. That simplifies the system constraint and coupling relationship, and reduces the complexity of the system design. An important principle for the system decomposition is to minimize the coupling between the various subsystems. As shown in Figure 4, the system has the coupling of three disciplines before adjusting, and is reduced to the coupling of the two disciplines after adjustment. That greatly reduces the calculating amount of the system. The MDO decomposition algorithms convert the system optimization problem into the main problem and subproblem sets according to the objective function, the constraints and the connection strength between them. 


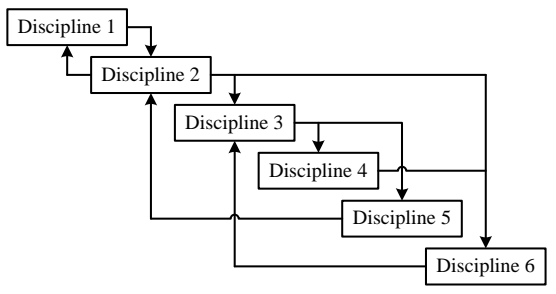

(a) Before decomposition

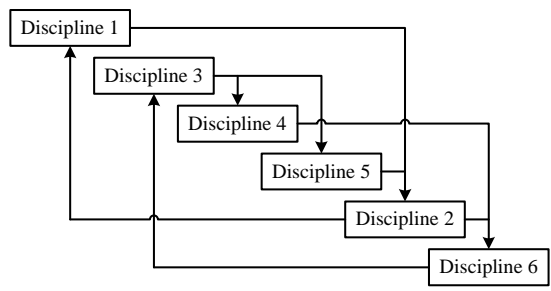

(b) After decomposition

Figure 4. The decomposition of the coupled system

In order to optimize, design and analyze the high-level band saw equipment fast and efficiently, the user-friendly multi-disciplinary design, optimization and analysis platform is developed using the multi-domain model of the band saw equipment and the multi-disciplinary design optimization tools (iSIGHT and ModelCenter). The platform has been practically applied in the development of the new products for the band saw equipment. That improves and perfects the design program of the band saw equipment to increase the efficiency and reliability of the design and development.

\section{REFERENCES}

[1] ZHU Jie, CHEN Guo-jin, WANG Wan-qiang, "Applying FPGA on Frequency Convert System of SVPWM," Mechanical \& Electrical Engineering Magazine, Vol. 22, No. 4, pp. 22-25, 2005.

[2] CHEN Guo-jin, YAO Zhai-rong, SUN Hong, "Realization of AC Motor Vector Control Based on DSP," Mechanical \& Electrical Engineering Magazine, Vol. 21, No. 6, pp. 27-30, 2004.

[3] CHEN Guo-jin, DENG Kai-ping, GONG You-ping, "The Design and Simulation of Walking Hydraulic Transmission System of Loader HT25J," Mechanical Engineer, No. 6, pp. 46-49, 2012.

[4] CHEN Guo-jin, ZHU Miao-fen, SU Shao-hui, GONG You-ping, ZHOU Da-da, "Research on the multi-domain modeling and optimizing method for loader executing system,” Proceedings-2010 International Conference on Intelligent System Design and Engineering Application, pp. 854-857, 2010.

[5] CHEN Guo-jin, DENG Kai-ping, GONG You-ping, "The Design and Simulation of Walking Hydraulic Transmission System of Loader HT25J,” Mechanical Engineer, No. 6, pp. 46-49, 2012. 